\title{
Gender-based violence and engagement in biomedical HIV prevention, care and treatment: a scoping review
}

\author{
Anna M. Leddy ${ }^{1 *}$, Ellen Weiss ${ }^{2}$ Eileen Yam $^{2}$ and Julie Pulerwitz ${ }^{2}$
}

\begin{abstract}
Background: While gender-based violence (GBV) has been shown to increase women's risk of HIV acquisition, the role of GBV in the HIV testing to care continuum is less clear. Clarifying how GBV may act as a barrier to accessing HIV services, treatment and care - such as anti-retroviral treatment (ART) or pre-exposure prophylaxis (PrEP) - will not only provide insights into how to best meet individual women's HIV care needs, but also inform public health oriented HIV epidemic control strategies.

Methods: Through a comprehensive scoping review, we synthesized and analyzed existing evidence regarding the influence of GBV on engagement in PrEP and the HIV care continuum among women living with HIV, including members of key populations (female sex workers, transgender women and women who use drugs). We explored PubMed, Scopus and Web of Science for peer-reviewed studies published in 2003-2017. Of the 279 sources identified, a subset of 51 sources met the criteria and were included in the scoping review.

Results: Studies were identified from 17 countries. The majority of studies utilized quantitative cross-sectional designs $(n=33)$, with the rest using longitudinal $(n=4)$, qualitative $(n=10)$ or mixed methods $(n=4)$ designs. Taken together, findings suggest that GBV impedes women's uptake of HIV testing, care, and treatment, yet this can vary across different geographic and epidemic settings. Substantial gaps in the literature do still exist, including studies on the impact of GBV on engagement in PrEP, and research among key populations.

Conclusions: This scoping review contributes to our knowledge regarding the role GBV plays in women's engagement in PrEP and the HIV care continuum. Findings reveal the need for more longitudinal research to provide insights into the causal pathways linking GBV and HIV care and treatment outcomes. Research is also needed to illuminate the impact of GBV on PrEP use and adherence as well as the impact of GBV on engagement along the HIV care continuum among key populations. It is critical that programs and research keep pace with these findings in order to reduce the global burden of GBV and HIV among women.
\end{abstract}

Keywords: Gender-based violence, Intimate partner violence, Pre-exposure prophylaxis, HIV care and treatment

\section{Background}

Gender-based violence (GBV), defined as violence perpetrated against an individual based on their gender/ gender identity [1, 2], is an important global health and human rights concern. GBV includes physical, sexual and psychological/emotional violence and can be perpetrated by a variety of actors, including intimate partners

\footnotetext{
* Correspondence: anna.leddy@ucsf.edu

${ }^{1}$ Division of Prevention Science, Center for AIDS Prevention Studies,

University of California, San Francisco, 550 16th St., 3rd Floor, San Francisco,

CA 94158, USA

Full list of author information is available at the end of the article
}

(referred to as intimate partner violence (IPV)), family members, community members, and representatives of the state (e.g. law enforcement officials) [1-3]. GBV is a common experience for women globally, with estimates suggesting that 1 in 3 women experience some form of GBV in their lifetime, primarily from an intimate partner [4]. Marginalized populations including female sex workers (FSW), transgender women, and women who use drugs experience even higher rates of GBV, often perpetrated by intimate partners and non-partners such as representatives of the state [5-7].

(C) The Author(s). 2019 Open Access This article is distributed under the terms of the Creative Commons Attribution 4.0 International License (http://creativecommons.org/licenses/by/4.0/), which permits unrestricted use, distribution, and 
GBV is associated with several acute and long-lasting health consequences [8], including HIV $[9,10]$. Globally, women are disproportionately affected by HIV-particularly in the epidemic's epicenter in sub-Saharan Africa-and HIV is the leading cause of death among women of reproductive age [11]. FSW, transgender women, and women who inject drugs are at even greater risk for HIV acquisition and HIV-related morbidity and mortality, due to their marginalized status in society, and the associated barriers they face in accessing HIV services [11]. Because of this, members of these populations have been identified as 'key populations' by international HIV organizations including the Joint United Nations Programme on HIV/AIDS (UNAIDS) [12].

A large body of evidence has demonstrated an inextricable link between GBV and HIV among women $[9,10]$. Women who experience GBV are more likely to engage in HIV risk behaviors such as condomless sex and are more likely to be living with HIV $[9,10,13]$. Similarly, evidence suggests that women living with HIV (WLHIV) are at increased risk for experiencing violence [14-16]. However, the role of GBV in the HIV care continuum (which includes HIV testing, and appropriate care and treatment for HIV or to prevent transmission via PrEP) is less clear. It is critical to clarify how GBV may act as a barrier to accessing HIV testing, linking to and staying engaged in HIV care and treatment, as well as PrEP, not only to address violence against individual women and meet their HIV care needs, but to also achieve public health-oriented HIV epidemic control goals. In 2015, for example, the World Health Organization (WHO) published guidelines which promoted the use of anti-retroviral treatment (ART) by anyone diagnosed with HIV, given the protective effects of early treatment initiation [17-19]. Further, in 2017, UNAIDS adopted the 'epidemic control' paradigm whereby the global HIV response is now working towards 90\% awareness of HIV status, $90 \%$ of those with HIV on treatment, and $90 \%$ of those on treatment virally suppressed [20]. Also in 2017, WHO finalized guidelines promoting pre-exposure prophylaxis (PrEP) - a formulation of antiretrovirals (ARVs) that prevents HIV acquisition even if exposed to HIV [21, 22] - for all those at substantial risk of HIV, including members of key populations [18]. In response, a massive global effort to encourage HIV testing and treatment has been rolled out, as testing is the entrypoint to HIV care and ART for those living with HIV, as well as PrEP for those at substantial risk of HIV. Key to the success of both HIV treatment and PrEP use is the regular adherence to the medications [23].

Over the past several years, a limited body of evidence has documented GBV as a barrier to women's engagement in the HIV care continuum. For example, a 2015 systematic review and meta-analysis by Hatcher and colleagues [24] - conducted before the guidelines mentioned above were established - explored the effects of IPV on ART use, ART adherence (measured via self-report and viral load), and retention in HIV care among WLHIV. The review identified 13 cross-sectional studies, primarily from the United States, and the meta-analysis demonstrated that IPV was associated with lower ART use, lower selfreported ART adherence, and lower odds of viral suppression [24]. This review did not include the literature exploring the effect of GBV on HIV testing or PrEP, and none of the identified studies included key populations. The authors noted the need for future research to explore the effect of GBV on the care continuum for these populations [24].

In light of the new global test and start guidelines [18], research is needed to summarize the evidence regarding the role GBV plays in engagement in the HIV testing to care continuum and PrEP among women, including members of key populations. Such a review can provide important insights into areas for future research and possible avenues for intervention. Accordingly, building upon the findings of the 2015 systematic review, we conducted an updated review of the evidence linking GBV to engagement in the HIV care continuum and PrEP using a 'scoping' methodology. A scoping review provides a more comprehensive review of the literature than a systematic review by looking broadly across study designs (for more detail, see the methods section) [25]. The present study aimed to expand upon the prior systematic review in two ways. First, we examined the evidence regarding the relationship between GBV and HIV testing as well as PrEP use and adherence, in addition to care and treatment. Second, we sought to identify studies that assessed the effect of GBV on engagement in the HIV care continuum and PrEP among members of key populations, including FSW, transgender women, and women who use drugs.

\section{Methods \\ Scoping review}

We conducted a scoping review, which enables researchers to summarize what is known about a certain topic for dissemination to policy makers and practitioners, and to identify gaps in the existing literature [25]. In contrast to systematic reviews, which are guided by a research question focused on a particular study design (typically restricted to quantitative methods), scoping reviews aim to "identify all relevant literature regardless of study design" $[25]^{(\mathrm{p} .22)}$. Additionally, scoping reviews call for an iterative process of refining search terms as the researcher becomes more familiar with the literature, to ensure the review is comprehensive [25].

Identifying the research questions and relevant literature This scoping review was guided by Arksey and O'Malley's (2005) methodological framework [25], and examined the 
known relationship between GBV and engagement in the HIV care continuum and PrEP among women, including members of key populations (FSW, female drug users, and transgender women). When examining the care continuum, we included HIV testing, linkage to and engagement in care, ART adherence and viral suppression. The team identified a search strategy based on a review of the literature and medical subject heading $(\mathrm{MeSH})$ terms. We explored the three search engines (PubMed, Scopus and Web of Science) for studies published in peer-reviewed journals in English between January 2003 and November 2017. We began our search in 2003 given that the WHO and UNAIDS began their initiative to roll out ART in low and middle income countries during that year [26]. Table 1 outlines the search terms used for each search engine. For each database, we conducted separate searches for each population given that individual searches in some cases yield different (and more) articles than a combined search.

\section{Selecting the literature}

We reviewed the titles and abstracts of all identified sources. The team created 'post hoc' exclusion criteria at this point to further narrow the review. Developing post-hoc exclusion criteria is a hallmark of the scoping review methodology. It is recommended to maximize the likelihood that researchers identify all relevant criteria as they familiarize themselves with the literature [25]. We excluded articles that were opinion pieces, protocols describing study designs, and literature reviews (although we did include individual studies that were referred to in literature reviews that met our inclusion criteria). We also excluded papers that explored violence

Table 1 Search terms

\begin{tabular}{|c|c|}
\hline $\begin{array}{l}\text { Search } \\
\text { engine }\end{array}$ & Search terms \\
\hline PubMed & $\begin{array}{l}\text { ((gender based violence [TIAB]) OR (intimate partner violence } \\
\text { [TIAB]) OR (violence against women [TIAB]) OR (domestic } \\
\text { violence [TIAB])) AND ((HIV services [TIAB]) OR (HIV care } \\
\text { cascade [TIAB]) OR (HIV treatment cascade [TIAB]) OR (HIV } \\
\text { care continuum [TIAB]) OR (Pre-exposure Prophylaxis [TIAB]) } \\
\text { OR (HIV test* [TIAB]) OR (linkage to HIV care* [TIAB]) OR } \\
\text { (engagement in HIV care* [TIAB]) OR (antiretroviral adherence } \\
\text { [TIAB]) OR (viral load [TIAB])) }\end{array}$ \\
\hline Scopus & $\begin{array}{l}\text { TITLE-ABS-KEY ("gender based violence" OR "intimate partner } \\
\text { violence" OR "violence against women" OR "domestic } \\
\text { violence") AND TITLE-ABS-KEY ("HIV services" OR"HIV care cas } \\
\text { cade" OR "HIV treatment cascade" OR "HIV care continuum" } \\
\text { OR "Pre-exposure prophylaxis" OR "HIV test*" OR "linkage to } \\
\text { HIV care*" OR "engagement in HIV care*" OR "antiretroviral ad } \\
\text { herence" OR "viral load") }\end{array}$ \\
\hline $\begin{array}{l}\text { Web of } \\
\text { Science }\end{array}$ & $\begin{array}{l}\text { TS = ("gender based violence" OR "intimate partner violence" } \\
\text { OR "violence against women" OR "domestic violence") AND } \\
\text { TS = ("HIV services" OR "HIV care cascade" OR "HIV treatment } \\
\text { cascade" OR "HIV care continuum" OR "Pre-exposure } \\
\text { prophylaxis" OR "HIV test*" OR "linkage to HIV care*" OR } \\
\text { "engagement in HIV care*" OR "antiretroviral adherence" OR } \\
\text { "viral load") }\end{array}$ \\
\hline
\end{tabular}

and only the acceptability or awareness of HIV services, as our focus was on the influence over behaviors. Finally, if papers included data from both male and female participants, we excluded those that did not disaggregate the results by sex. The articles of the remaining sources were reviewed in full. Reference sections of all included sources were hand searched for additional relevant sources not already identified by the database search. Relevant sources were included in the full review.

\section{Charting, collating and summarizing the information}

The first author created a matrix to chart relevant information about all the sources reviewed. Specifically, the chart included details about the study design, sample size, population and relevant findings. In accordance with Arksey and O'Malley's framework [25], the research team held meetings to discuss the overall themes emerging from the reviewed literature and to identify gaps in the literature that warranted further exploration.

\section{Results}

As shown in Fig. 1, the team identified 226 non-duplicate sources to review from the database search. An additional 53 sources were added after reviewing the reference sections of the sources identified by the database search. Of these 279 sources, a subset of 51 sources were included in the scoping review (Table 2). Studies were from 17 countries: 10 countries from Africa (Uganda, Kenya, Zambia, Malawi, Ethiopia, South Africa, Tanzania, Cameroon, Tunisia and Cote D'Ivoire), three countries from Asia (Nepal, India, Malaysia), two countries from South America (Dominican Republic and Bolivia), and North America (United States (U.S.) and Canada). The majority of studies identified utilized quantitative cross-sectional designs $(n=$ 33), four used quantitative longitudinal designs, ten were qualitative and four were mixed methods. Below, we outline the evidence regarding the effects of experiences of violence on women's engagement in the HIV care continuum and PrEP.

\section{GBV and its implications for engagement in the HIV care continuum and PrEP HIV testing}

The review yielded 19 quantitative studies (three longitudinal and 16 cross-sectional studies), one qualitative study and one mixed method study that explored the relationship between violence and HIV testing among women. Three studies were conducted among members of key populations (two studies were among women who use drugs and one was among FSW). We did not find any studies that examined the relationship between GBV and HIV testing among transgender women.

Results were mixed. A number of the studies found that experiences of violence were associated with reduced HIV 


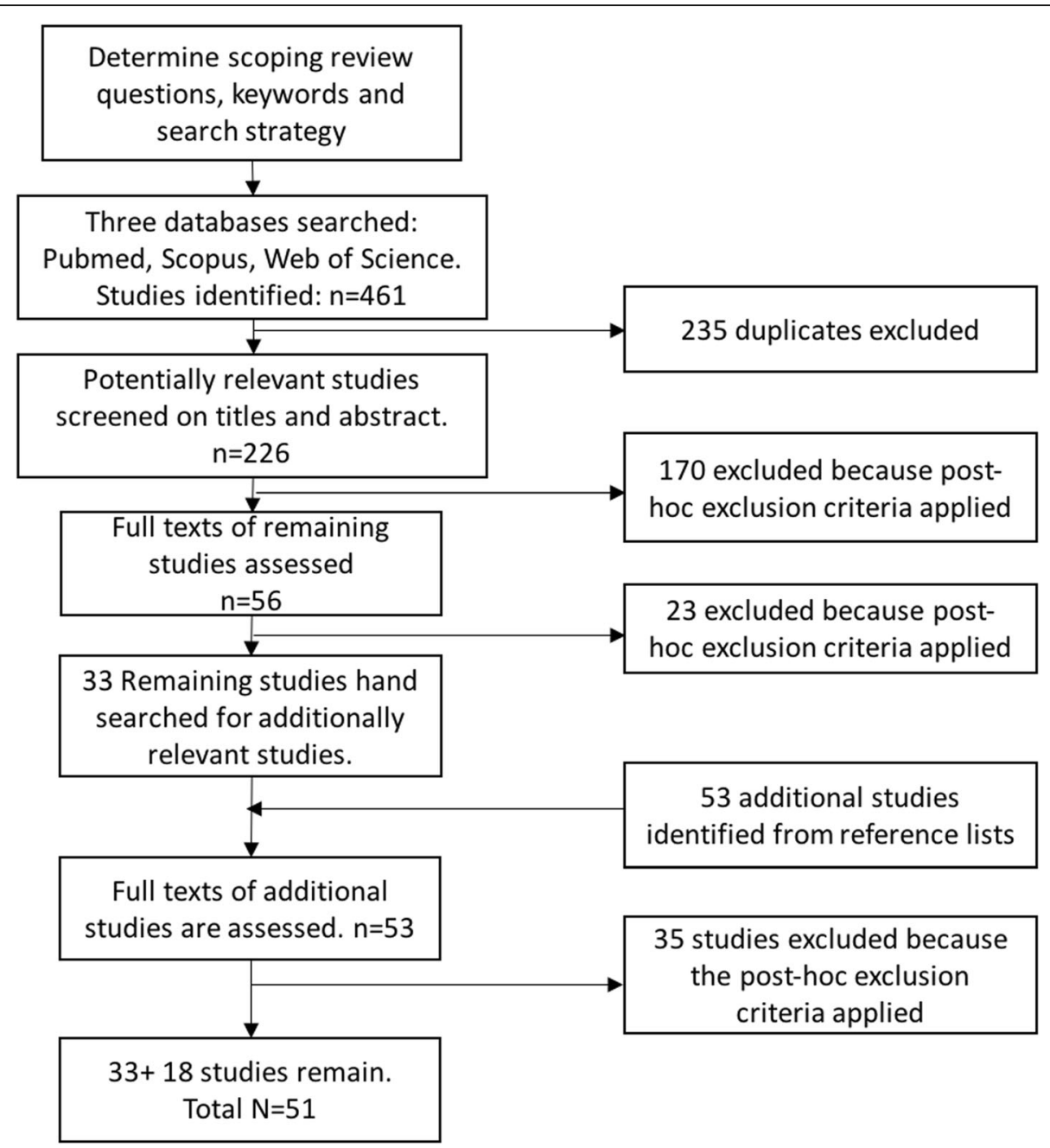

Fig. 1 Flow diagram of review process

testing among women [30, 33, 34, 36, 39]. Qualitative studies described how fear of a violent reaction from one's partner in the event of a positive test result contributed to reduced rates of HIV testing [43, 44]. A cross-sectional study by Turan et al. (2011) supported these findings by demonstrating that anticipated stigma (defined as break-up of marriage/relationship and physical violence from a partner) upon testing positive for HIV was associated with refusing to test for HIV [27].

Two studies among key populations also found a negative relationship between GBV and HIV testing [39, 65]. A cross-sectional study among women who use drugs in Malyasia found that experiences of adulthood violence from a partner were associated with failure to test for HIV [39]. Another study among substance-using black South African women found that those who experienced physical violence were less likely to be aware of their HIV-positive status [65].

At the same time, five additional studies, all from the U.S., found experiences of violence to be associated with increased HIV testing [31, 35, 37, 38, 40]. One of these studies was a longitudinal study, which found that experiences of intimate partner violence (IPV) in the past 12 months at baseline was significantly associated with increased odds of receiving a test for sexually transmitted infections (STIs) (including HIV) during a two-year follow up period [40].

Seven studies found no significant relationship between experiences of violence and uptake of HIV testing among women $[28,29,32,41,42,44,62]$. Two of these studies utilized longitudinal designs. Conroy et al. (2015) found that physical and sexual violence at baseline was not significantly associated with receiving a subsequent HIV test during a 16month follow up period among women in heterosexual couples in Malawi [41]. A cross-sectional study among FSW in Côte d'Ivoire also found no significant relationship between physical or sexual violence and uptake of HIV testing [62]. 


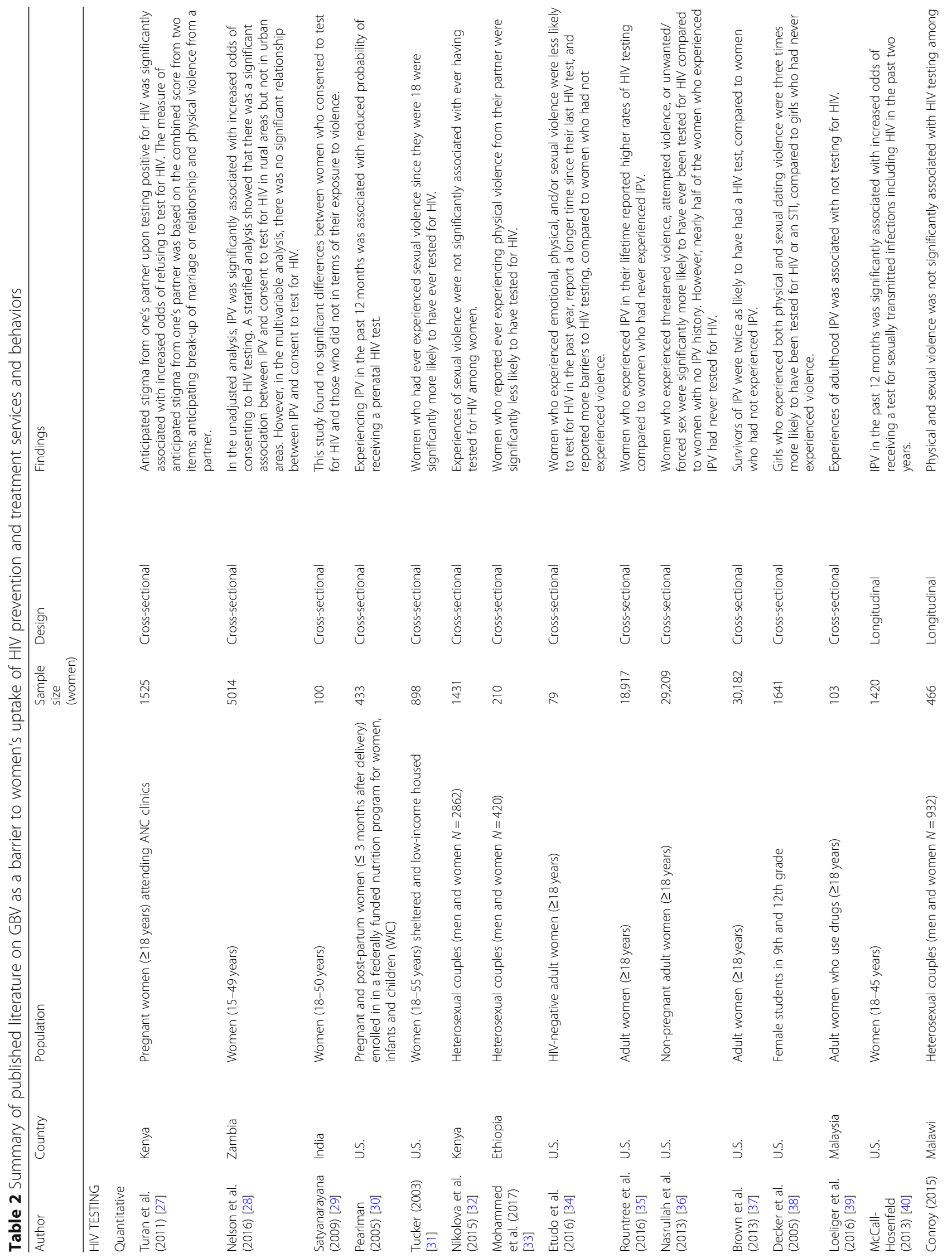




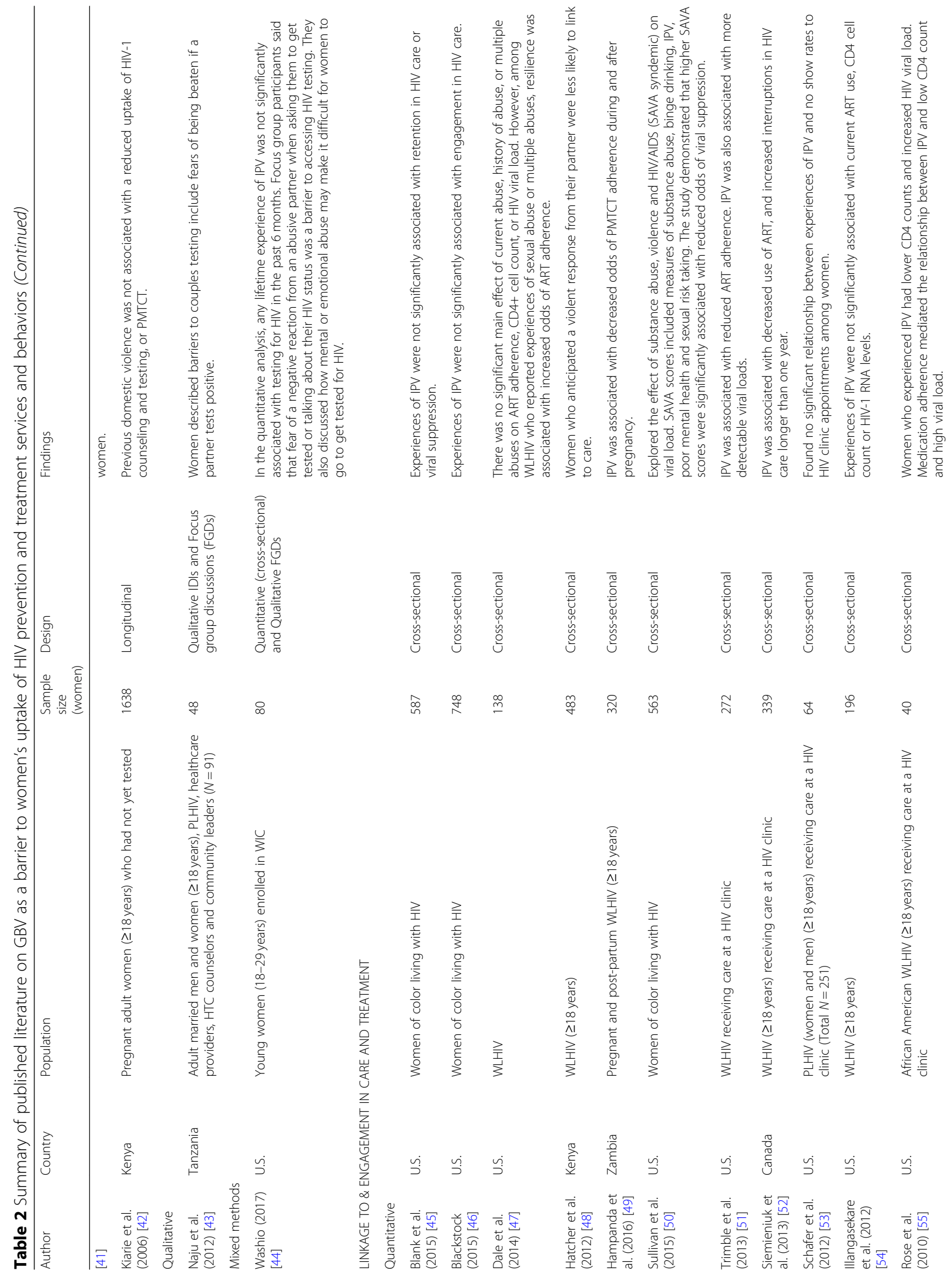




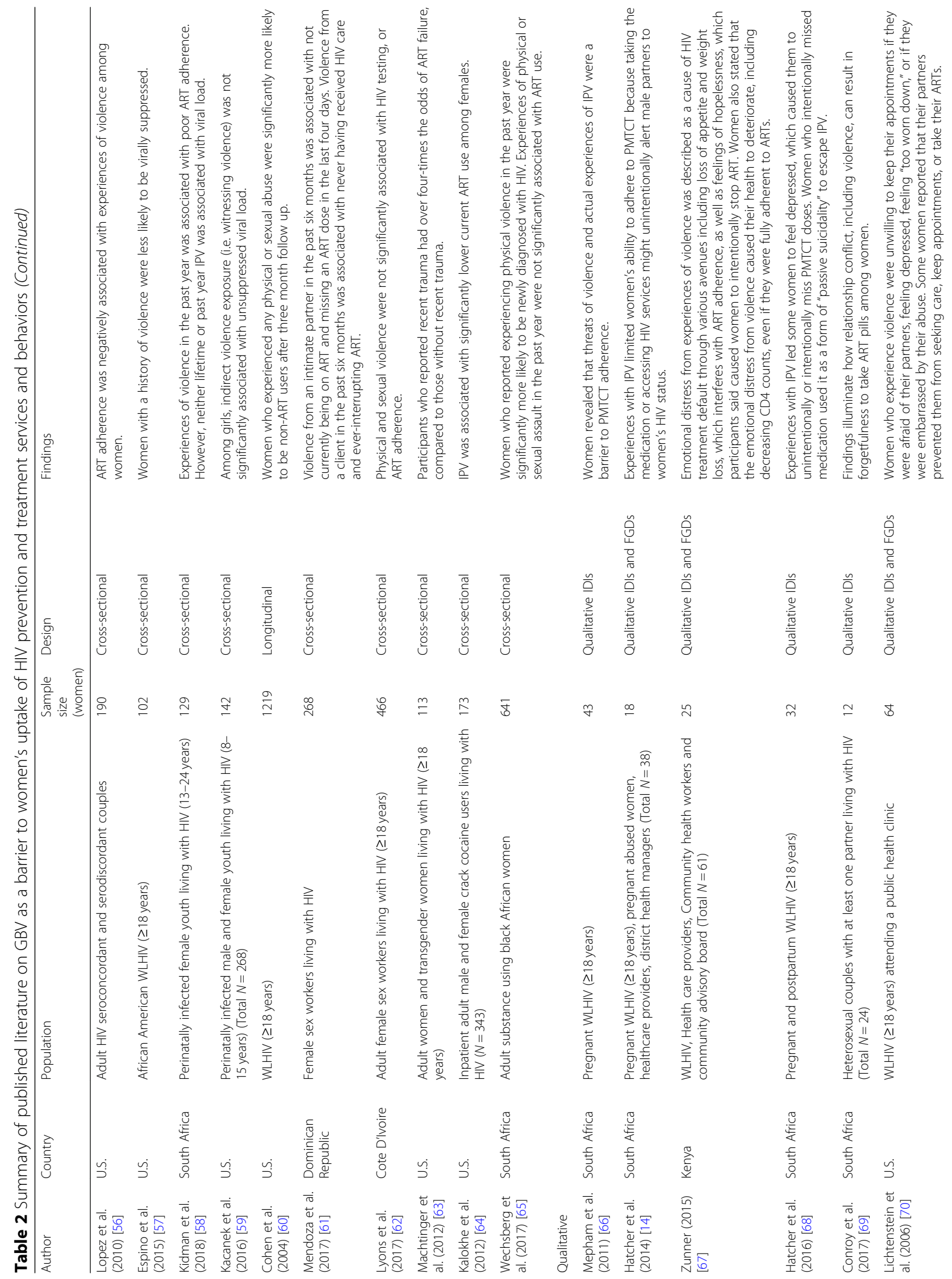




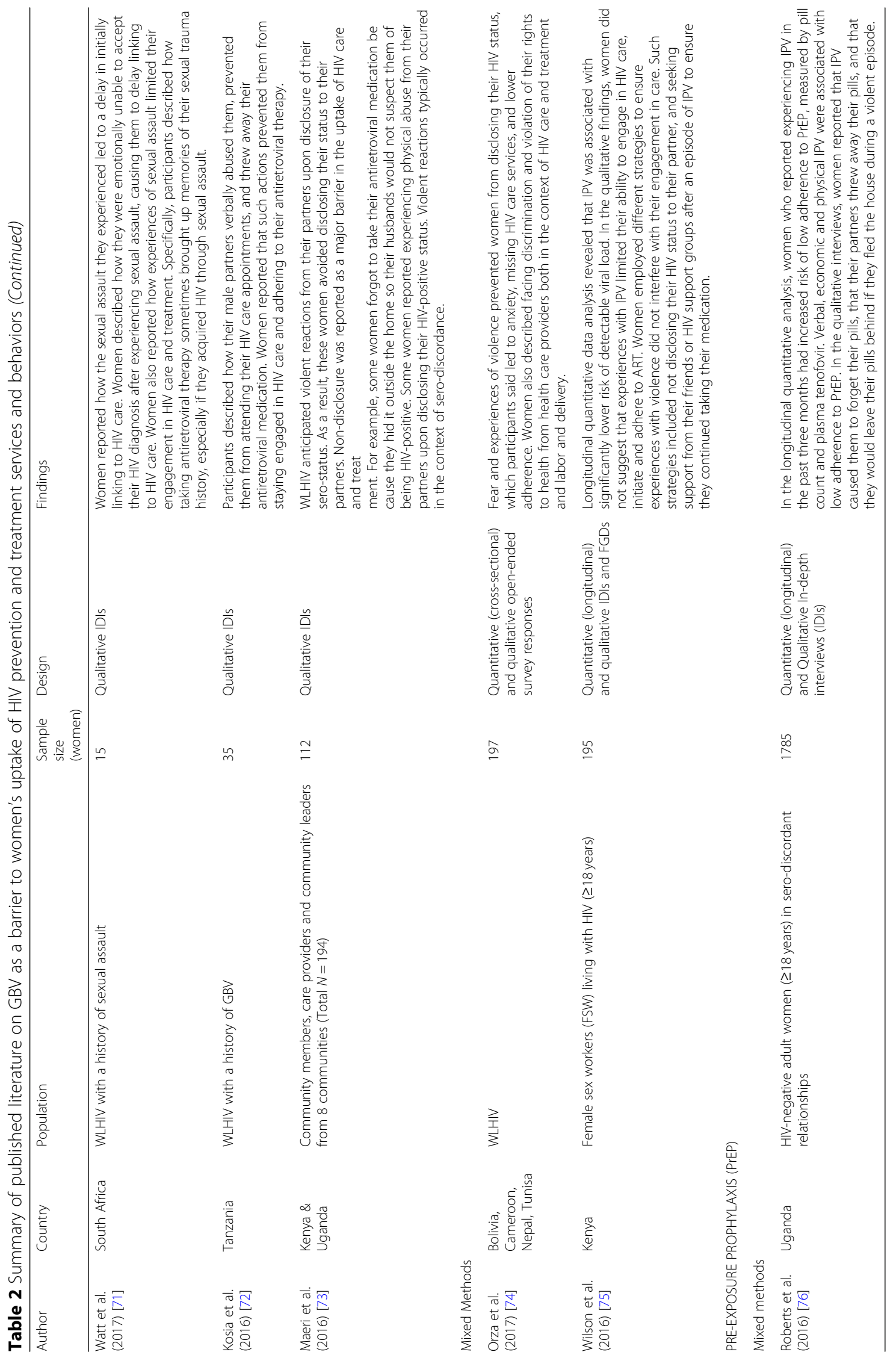




\section{Linkage to and engagement in HIV care}

The review yielded thirteen studies (six quantitative, five qualitative, and two mixed-methods studies) that explored the relationship between GBV and linkage to and engagement in HIV care among WLHIV. Two of these studies were conducted among FSW. We did not find any studies that examined this relationship among women who use drugs, or transgender women.

The research suggests that experiences of violence are associated with reduced linkage to HIV care among WLHIV [48, 71, 73], including FSW [61]. A cross-sectional study among WLHIV in Kenya found that women were less likely to link to HIV care if they anticipated a violent reaction from their partner upon learning the woman's HIV-positive serostatus [48]. This was supported by qualitative research from Uganda and Kenya, which found that women avoided disclosing their HIV-positive status to their partner because they feared a violent reaction [73]. Women revealed that non-disclosure was a major barrier to uptake of HIV care because they did not want to inadvertently disclose their status to their partner by seeking care [73].

There is also some evidence to suggest that GBV prevents WLHIV from staying engaged in HIV care, once they have already enrolled [52, 70-72]. A cross-sectional study among WLHIV in Canada found that experiences of IPV were associated with increased interruptions in HIV care longer than one year [52]. Qualitative evidence suggests that women skip their HIV care appointments due to fear that attending such appointments will unintentionally alert their partner to their HIV-positive status and result in violence [70]. Having partners who threaten women with violence or prevent them from attending their HIV care appointments may also prevent WLHIV from staying engaged in HIV care [70, 72]. Furthermore, women who experience violence may miss their appointments due to depression, physical illness, or injury caused by violence, and shame of being abused [70,71].

Three other studies found no significant relationship between experiences of violence and engagement in HIV care among WLHIV $[45,46,53]$. All three studies were cross-sectional and from the U.S. Additionally, a mixed methods study among FSW in Kenya found that GBV did not limit women's engagement in HIV care [75]. Findings from this study suggest that women utilized a number of different strategies to stay engaged in HIV care including not disclosing their HIV status to their partner and seeking support from their friends.

\section{Antiretroviral therapy initiation and adherence}

We identified 29 studies that explored the relationship between GBV and ART initiation and adherence among WLHIV. Eighteen studies were quantitative (17 crosssectional and one longitudinal), 9 were qualitative, and two utilized mixed methods. Six of these studies were conducted among members of key populations: three among FSW [61, 62, 75], two among women who use drugs [50, 64], and one among transgender women [63].

Taken together, evidence suggests that WLHIV who experience violence are significantly less likely to initiate [60] and adhere to ART [14, 49, 51, 52, 56, 58, 61, 64, $66,68,69,72,73]$, and ultimately achieve viral suppression [50, 51, 55, 57, 63]. In terms of ART initiation, a longitudinal study among WLHIV in the U.S. found that women who experienced physical or sexual violence were significantly more likely to be non-ART users after a three-month follow-up period [60]. In a cross-sectional study, Espino et al. (2015) found that African American women in the U.S. with a history of violence were significantly less likely to be virally suppressed than women without a history of violence [57]. Hampanda et al. (2016) found that violence from a partner was associated with reduced adherence to PMTCT during and after pregnancy among pregnant and post-partum WLHIV in Zambia, also assessed cross-sectionally [49].

When looking specifically at key populations, a crosssectional study among FSW living with HIV in the Dominican Republic found that experiencing violence from a non-paying intimate partner was associated with not currently being on ART and missing a recent ART dose [61]. Kalokhe et al. (2012) found that experiences of IPV was associated with significantly lower current ART use among female crack cocaine users in the U.S. [64]. Another study found that women of color from the U.S. with higher levels of substance abuse, binge drinking, IPV, poor mental health, and sexual risk taking had reduced odds of viral suppression [50]. Finally, Machtinger et al. (2012) found that recent trauma (defined as having been abused, threatened, or the victim of violence in the past 30 days) was associated with having a detectable viral load among both cis-gendered and transgender women [63] .

Qualitative studies shed light on potential mechanisms through which GBV can lead to poor ART adherence. Evidence suggests that women may choose to keep their HIV-positive status a secret from their partner because they fear their partner may become violent upon learning their HIV status [14, 73, 66, 68, 74]. As a consequence, women hide their pills and have to take their medication in secret $[14,68,73,74]$. This sometimes leads to missed doses of ART [14, 73, 74]. Additional qualitative research has revealed that some women's partners throw away their ART medication, or otherwise prevent them from taking their ART medication, which limits their adherence [70, 72]. Other research has demonstrated that WLHIV who experience violence from their partners can skip treatment due to depression or feelings of hopelessness [67-69].

Although the majority of evidence in the literature suggests a negative association between violence and ART use and adherence, we did find six studies among 
WLHIV that did not follow this trend $[45,46,54,59,62$, 75]. For example, a cross-sectional study of WLHIV attending an HIV clinic in Baltimore, Maryland did not find a significant association between GBV and current ART use, CD4 cell count, or HIV-1 RNA levels (which were used as a proxy for ART adherence) [54]. The authors argue that this finding may be due the fact that women in the sample were recruited from an HIV clinic and, therefore, all participants were engaged in HIV care and treatment [54]. As another example, a mixed methods study among FSW living with HIV in Kenya found that GBV was associated with significantly lower risk of a detectable viral load [75]. Women participating in the qualitative component described how they used different strategies, such as keeping their status secret from their abusive partner, to ensure that they were not prevented from accessing care [75]. Additionally, a cross-sectional study among FSW in Cote D'Ivoire did not find a statistically significant relationship between experiences of violence (physical or sexual) and ART adherence [62].

\section{Pre-exposure prophylaxis (PrEP)}

We found only one study that examined the relationship between GBV and PrEP use and adherence. Roberts et al. (2016) conducted a mixed-methods study among serodiscordant couples in Uganda, which found that HIVnegative women who experienced IPV in the past three months had a significantly increased risk of low adherence to PrEP by pill count and by plasma tenofovir, compared to women who had not experienced violence [76]. In qualitative interviews, HIV-negative women described how conflict in their homes made it difficult for them to remember to take their PrEP pills [76]. Others reported escaping their homes after a violent episode and forgetting to take their PrEP pills with them, and, as a result, they missed some doses [76].

We were unable to find studies that examined the relationship between experiences of violence and PrEP use among FSW, transgender women, or women who use drugs.

\section{Discussion}

Taken together, findings from this wide-ranging examination of recent literature suggest that GBV impedes women's engagement in biomedical HIV prevention, care, and treatment services. We see a similar relationship for female members of key populations, with a small but growing evidence base. Several studies suggest that women who experience violence are less likely to link to HIV care $[48,61,71,73]$, initiate and adhere to ART $[14,49,51,52,56,58,60,61,64,66,68,69]$ and less likely to achieve viral suppression $[50,51,55,57$, 63]. Qualitative evidence suggests women avoid disclosing their HIV-positive status to their partner because they fear their partner may become violent upon learning their HIV status $[14,66,68,73,74]$. Such nondisclosure, was highlighted as a barrier to engagement in HIV care and treatment, due to fear of inadvertently alerting one's partner to one's HIV positive status, and potentially experiencing violence $[14,68,73,74]$. Other research suggests a mental health pathway linking experiences of GBV to sub-optimal engagement in HIV care and treatment [67-69].

However, evidence specifically regarding the relationship between GBV and uptake of HIV testing varied across settings. Several studies from high-income countries, for example, found that GBV was associated with increased testing [31, 35-38, 40], while most studies from low and middle-income countries revealed no significant relationship between GBV and HIV testing [28, $29,32,41,42]$. While these studies were for the most part cross-sectional, and therefore do not indicate whether experiences of GBV led to increased testing or vice versa, it is feasible that the positive relationship between experiences of GBV and HIV testing found in high-income countries may in fact reflect increased perceived risk of HIV among survivors [77] and an interest in ascertaining HIV status, as well as enhanced infrastructure in these settings to address and respond to GBV.

There were several notable remaining gaps in the literature. We only found one study that examined the relationship between experiences of GBV and engagement in PrEP [76]. This study was from Uganda, and there were no such studies from higher-income countries, where PrEP is more accessible and established as an HIV prevention strategy. Additional research is needed, including longitudinal studies, to further understand the role that GBV plays in engagement in PrEP. The need for such evidence will become increasingly pronounced as PrEP is introduced and scaled up in the Global South.

Information about the effects of GBV on engagement along the HIV care continuum for female key populations living with HIV was also limited, and thus additional research is sorely needed. Furthermore, we did not find any study that explored the relationship between GBV and PrEP use among female key populations. It is critical to understand how experiences of GBV may influence these populations' access to PrEP, especially given their heightened risk for both GBV and HIV [6, 7, 11, 78], and the $2017 \mathrm{WHO}$ guidelines highlighting the need for PrEP among these populations [18].

It is also important to note the dearth of longitudinal studies that have explored the relationship between GBV and women's engagement in biomedical HIV prevention, care and treatment. This review identified only four longitudinal studies. Three of these studies examined the relationship between GBV and HIV testing, and one 
assessed the effect of GBV on ART use. We did not identify any longitudinal studies that examined the relationship between experiences of GBV and linkage to and engagement in HIV care as well as PrEP use or adherence. Longitudinal studies are urgently needed to better understand the effect of GBV on engagement in biomedical HIV prevention, care and treatment to inform future intervention work.

Given the existing evidence highlighted in this review suggesting that GBV is an important barrier to engagement in the HIV care continuum, programmatic responses are urgently needed. However, our review only identified one paper describing an evaluation of a program that explicitly sought to address GBV with the goal of improving any HIV care continuum outcomes [79]. Collins et al. (2017) used a quasi-experimental design to assess the effects of a muti-component intervention, "Creating Lasting Family Connections," among African American women in the U.S. [79]. This intervention sought to address factors that place African American women at increased risk for HIV including substance use and violence. Intervention modules promoted positive relationship and parenting skills, conflict resolution skills, offered guidance on how to incorporate substance use and violence prevention messages into activities with children, and improved knowledge about HIV transmission and substance abuse. Participants in the intervention were significantly more likely to test for HIV, and significantly less likely to report IPV in the past 3 months, relative to the comparison group [79]. We did not come across literature describing evaluations that found effects on engagement in HIV care or treatment, or uptake and adherence to PrEP among HIV negative women.

We do acknowledge that a number of studies have evaluated the effects of interventions that have sought to address GBV and HIV risk [80-83]. Researchers could draw upon related lessons learned to address GBV and engagement in HIV care and treatment. For example, several past interventions have aimed to mitigate and transform inequitable gender dynamics (such as support for 'toxic' masculinities, and unequal power in relationships) that can lead to GBV and HIV risk behaviors [81, 83, 84]. Future research could explore the impact of promoting gender equitable norms on both GBV and engagement in the HIV care continuum and PrEP. Other researchers have integrated GBV screening into HIV testing services to identify survivors of violence, who might otherwise not report their experience, and offer support and information $[85,86]$. It is also possible that HIV care appointments could provide an important opportunity for health care providers or counselors to screen women for GBV and strategize ways to prevent future violence and stay engaged in HIV care and treatment.

This scoping review was limited to articles written in English, and as such we may have excluded relevant articles written in other languages. Additionally, while we recognize that both women and men experience $\mathrm{GBV}$, this review is focused on violence experienced by women, and the HIV service experiences of women. As such, this study does not address what is known about GBV and engagement in the HIV care continuum and PrEP among men, including men who have sex with men. This area certainly warrants further research.

\section{Conclusion}

The linkages between GBV and HIV acquisition have been documented since the early 2000s. This review presents the latest evidence on how GBV can also impede the uptake of HIV testing, care and treatment and PrEP. Findings suggest that the relationship between experiences of GBV and sub-optimal engagement in the HIV care continuum is also significant, although it can vary by geographic context and epidemic setting. However, this review highlighted important gaps in the literature including a dearth of research on the role GBV plays in PrEP use and adherence, limited research on the effect of GBV on engagement in HIV care and treatment and PrEP among members of key populations, and very few longitudinal studies. Future research should prioritize addressing these gaps in the literature.

The global HIV response continues to evolve at an extraordinary pace, with new biomedical strategies creating the potential for epidemic control on the foreseeable horizon. It is critical, however, that programs and research keep pace with these changes by continuing to train a critical lens on gender inequity-and GBV as a particularly severe sequela-as a persistent driver of HIV. Only by continuing to place women at the center of the global HIV response will we achieve the ambitious UNAIDS HIV epidemic control goals.

\section{Abbreviations}

ART: Antiretroviral therapy; ARVs: Antiretrovirals; FSW: Female sex workers; GBV: Gender based violence; IPV: Intimate partner violence; PrEP: Preexposure prophylaxis; U.S.: United States; UNAIDS: Joint United Nations Program on HIV/AIDS; WHO: World Health Organization; WLHIV: Women living with HIV

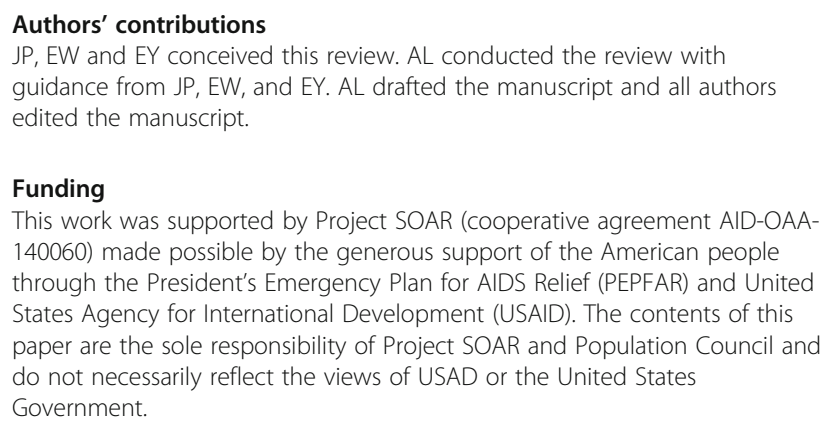

Availability of data and materials Not applicable. 


\section{Ethics approval and consent to participate}

Not applicable.

\section{Consent for publication}

Not applicable.

\section{Competing interests}

The authors declare that they have no competing interests.

\section{Author details}

'Division of Prevention Science, Center for AIDS Prevention Studies, University of California, San Francisco, 550 16th St., 3rd Floor, San Francisco, CA 94158, USA. ²Population Council, 4301 Connecticut Ave. NW, \# 280, Washington, DC 20008, USA

Received: 7 January 2019 Accepted: 17 June 2019 Published online: 08 July 2019

\section{References}

1. USAID. United States strategy to prevent and respond to gender-based violence globally: 2016 update. Washington, D.C; 2016

2. United Nations. Declaration on the elimination of violence against women 1993

3. Interagency Standing Committee. Guidelines for gender-based violence interventions in humanitarian settings. Geneva: the IASC taskforce on gender in humanitarian assistance; 2005.

4. World Health O. Global and regional estimates of violence against women: prevalence and health effects of intimate partner violence and non-partner sexual violence: WHO; 2013. Contract No.: Report

5. Wirtz AL, Poteat TC, Malik M, Glass N. Gender-based violence against transgender people in the United States: a call for research and programming. Trauma Violence Abuse. 2018:1524838018757749.

6. El-Bassel N, Terlikbaeva A, Pinkham S. HIV and women who use drugs: double neglect, double risk. Lancet. 2010;376(9738):312-4

7. Deering KN, Amin A, Shoveller J, Nesbitt A, Garcia-Moreno C, Duff P, et al. A systematic review of the correlates of violence against sex workers. Am J Public Health. 2014;104:e42-54.

8. Campbell JC. Health consequences of intimate partner violence. Lancet. 2002;359(9314):1331-6

9. Dunkle KL, Decker MR. Gender-based violence and HIV: reviewing the evidence for links and causal pathways in the general population and high-risk groups. Am J Reprod Immunol. 2013:69(Suppl 1):20-6 (New York, NY: 1989).

10. Li Y, Marshall CM, Rees HC, Nunez A, Ezeanolue EE, Ehiri JE. Intimate partner violence and HIV infection among women: a systematic review and metaanalysis. J Int AIDS Soc. 2014;17:18845.

11. UNAIDS. Global AIDS Update Geneva; 2018

12. UNAIDS. Key populations 2019 [Available from: http://www.unaids.org/en/ topic/key-populations.

13. Maman S, Mbwambo JK, Hogan NM, Kilonzo GP, Campbell JC, Weiss E, et al. HIV-positive women report more lifetime partner violence: findings from a voluntary counseling and testing clinic in Dar Es Salaam, Tanzania. Am J Public Health. 2002:92(8):1331-7.

14. Hatcher AM, Woollett N, Pallitto CC, Mokoatle K, Stockl H, MacPhail C, et al. Bidirectional links between HIV and intimate partner violence in pregnancy: implications for prevention of mother-to-child transmission. J Int AIDS Soc. 2014:17:19233.

15. Olowookere SA, Fawole OI, Adekanle DA, Adeleke NA, Abioye-Kuteyi EA. Patterns and correlates of intimate partner violence to women living with HIV/AIDS in Osogbo, Southwest Nigeria. Violence Against Women. 2015:21(11):1330-40.

16. Mulrenan C, Colombini M, Howard N, Kikuvi J, Mayhew SH, Integra I. Exploring risk of experiencing intimate partner violence after HIV infection: a qualitative study among women with HIV attending postnatal services in Swaziland. BMJ Open. 2015;5(5):e006907.

17. Cohen MS, Chen YQ, McCauley M, Gamble T, Hosseinipour MC, Kumarasamy N, et al. Antiretroviral therapy for the prevention of HIV-1 transmission. N Engl J Med. 2016:375(9):830-9.

18. World Health O. Consolidated guidelines on the use of antiretroviral drugs for treating and preventing HIV infection: recommendations for a public health approach: Contract No.: Report; 2016.

19. Montaner JS, Lima VD, Harrigan PR, Lourenco L, Yip B, Nosyk B, et al. Expansion of HAART coverage is associated with sustained decreases in
HIV/AIDS morbidity, mortality and HIV transmission: the "HIV treatment as prevention" experience in a Canadian setting. PLoS One. 2014;9(2):e87872.

20. UNAIDS. Making the end of AIDS real. Consensus building around what we mean by "epidemic control". Switzerland: Glion; 2017.

21. Baeten JM, Donnell D, Ndase P, Mugo NR, Campbell JD, Wangisi J, et al. Antiretroviral prophylaxis for HIV prevention in heterosexual men and women. N Engl J Med. 2012;367(5):399-410.

22. McCormack S, Dunn DT, Desai M, Dolling DI, Gafos M, Gilson R, et al. Preexposure prophylaxis to prevent the acquisition of HIV-1 infection (PROUD): effectiveness results from the pilot phase of a pragmatic open-label randomised trial. Lancet (London, England). 2016;387(10013):53-60.

23. World Health O. Antiretroviral treatment as prevention (TasP) of HIV and TB. Geneva: WHO; 2012.

24. Hatcher AM, Smout EM, Turan JM, Christofides N, Stockl H. Intimate partner violence and engagement in HIV care and treatment among women: a systematic review and meta-analysis. AIDS (London, England). 2015.

25. Arksey H, O'Malley L. Scoping studies: towards a methodological framework. Int J Social Research Methodology. 2005;8(1):19-32.

26. Weller I. Delivery of antiretroviral therapy in sub-saharan Africa. Clin Infect Dis. 2006;43(6):777-8.

27. Turan JM, Bukusi EA, Onono M, Holzemer WL, Miller S, Cohen CR. HIV/AIDS stigma and refusal of HIV testing among pregnant women in rural Kenya: results from the MAMAS study. AIDS Behav. 2011;15(6):1111-20.

28. Nelson KA, Ferrance JL, Masho SW. Intimate partner violence, consenting to HIV testing and HIV status among Zambian women. Int J STD AIDS. 2016;27(10):832-9.

29. Satyanarayana VA, Chandra PS, Vaddiparti K, Benegal V, Cottler LB. Factors influencing consent to HIV testing among wives of heavy drinkers in an urban slum in India. AIDS Care. 2009;21(5):615-21.

30. Pearlman DN, Averbach AR, Zierler S, Cranston K. Disparities in prenatal HIV testing: evidence for improving implementation of CDC screening guidelines. J Natl Med Assoc. 2005:97(7 Suppl):44S-51S.

31. Tucker JS, Wenzel SL, Elliott MN, Hambarsoomian K, Golinelli D. Patterns and correlates of HIV testing among sheltered and low-income housed women in Los Angeles County. J Acquir Immune Defic Syndr. 2003;34(4):415-22.

32. Nikolova SP, Small E, Mengo C. Components of resilience in gender: a comparative analysis of HIV outcomes in Kenya. Int J STD AIDS. 2015;26(7):483-95.

33. Mohammed BH, Johnston JM, Harwell $\mathrm{Jl}, \mathrm{Yi}$ H, Tsang KW, Haidar JA. Intimate partner violence and utilization of maternal health care services in Addis Ababa, Ethiopia. BMC Health ServRes. 2017:17:178-88.

34. Etudo O, Metheny N, Stephenson R, Kalokhe AS. Intimate partner violence is linked to less HIV testing uptake among high-risk, HIV-negative women in Atlanta. AIDS Care. 2017:1-4

35. Rountree MA, Chen L, Bagwell M. HIV-testing rates and testing locations among women who have experienced intimate partner violence: data from the centers for disease control behavioral risk factor surveillance system, 2006. Violence Against Women. 2016:22(4):399-414.

36. Nasrullah M, Oraka E, Breiding MJ, Chavez PR. HIV testing and intimate partner violence among non-pregnant women in 15 US states/territories: findings from behavioral risk factor surveillance system survey data. AIDS Behav. 2013;17(7):2521-7.

37. Brown MJ, Weitzen S, Lapane KL. Association between intimate partner violence and preventive screening among women. Journal of women's health (2002). 2013:22(11):947-52

38. Decker MR, Silverman JG, Raj A. Dating violence and sexually transmitted disease/HIV testing and diagnosis among adolescent females. Pediatrics. 2005;116(2):e272-6.

39. Loeliger KB, Marcus R, Wickersham JA, Pillai V, Kamarulzaman A, Altice FL. The syndemic of HIV, HIV-related risk and multiple co-morbidities among women who use drugs in Malaysia: important targets for intervention. Addict Behav 2016:53:31-9.

40. McCall-Hosenfeld JS, Chuang CH, Weisman CS. Prospective association of intimate partner violence with receipt of clinical preventive services in women of reproductive age. Womens Health Issues. 2013;23(2):e109-16.

41. Conroy AA. The influence of relationship power dynamics on HIV testing in rural Malawi. J Sex Res. 2015;52(3):347-59.

42. Kiarie JN, Farquhar C, Richardson BA, Kabura MN, John FN, Nduati RW, et al. Domestic violence and prevention of mother-to-child transmission of HIV-1. AIDS (London, England). 2006:20(13):1763-9.

43. Naju B, Watt MH, Ostermann J, Manongi R, Sikkema KJ. Percieved acceptability of home-based couples voluntary HIV counseling and testing in northern Tanzania. AIDS Care. 2012;24(4):413-9. 
44. Washio Y, Wright EN, Flores D, Davis A, Chittams J, Anagnostopulos C, et al. Perspectives on HIV testing among WIC-enrolled postpartum women: implications for intervention development. AIDS Educ Prev. 2017;29(5):457-74.

45. Blank AE, Fletcher J, Verdecias N, Garcia I, Blackstock O, Cunningham C. Factors associated with retention and viral suppression among a cohort of HIV+ women of color. AIDS Patient Care STDs. 2015;29(Suppl 1):S27-35.

46. Blackstock OJ, Blank AE, Fletcher JJ, Verdecias N, Cunningham CO. Considering care-seeking behaviors reveals important differences among HIV-positive women not engaged in care: implications for intervention. AIDS Patient Care STDs. 2015;29(Suppl 1):S20-6.

47. Dale S, Cohen M, Weber K, Cruise R, Kelso G, Brody L. Abuse and resilience in relation to HAART medication adherence and HIV viral load among women with HIV in the United States. AIDS Patient Care STDs. 2014;28(3):136-43.

48. Hatcher AM, Turan JM, Leslie HH, Kanya LW, Kwena Z, Johnson MO, et al. Predictors of linkage to care following community-based HIV counseling and testing in rural Kenya. AIDS Behav. 2012;16(5):1295-307.

49. Hampanda KM. Intimate partner violence and HIV-positive women's nonadherence to antiretroviral medication for the purpose of prevention of mother-to-child transmission in Lusaka, Zambia. Social science \& medicine (1982). 2016;153:123-30.

50. Sullivan KA, Messer LC, Quinlivan EB. Substance abuse, violence, and HIV/ AIDS (SAVA) syndemic effects on viral suppression among HIV positive women of color. AIDS Patient Care STDs. 2015;29(Suppl 1):S42-8.

51. Trimble DD, Nava A, McFarlane J. Intimate partner violence and antiretroviral adherence among women receiving care in an urban southeastern Texas HIV clinic. J Assoc Nurses AIDS Care. 2013;24(4):331-40

52. Siemieniuk RA, Krentz HB, Miller P, Woodman K, Ko K, Gill MJ. The clinical implications of high rates of intimate partner violence against HIV-positive women. Journal of acquired immune deficiency syndromes (1999). 2013;64(1):32-8.

53. Schafer KR, Brant J, Gupta S, Thorpe J, Winstead-Derlega C, Pinkerton R, et al. Intimate partner violence: a predictor of worse HIV outcomes and engagement in care. AIDS Patient Care STDs. 2012;26(6):356-65.

54. Illangasekare S, Tello M, Hutton H, Moore R, Anderson J, Baron J, et al. Clinical and mental health correlates and risk factors for intimate partner violence among HIV-positive women in an inner-city HIV clinic. Womens Health Issues. 2012;22(6):e563-e9.

55. Rose RC, House AS, Stepleman LM. Intimate partner violence and its effects on the health of African American HIV-positive women. Psychol Trauma Theory Res Pract Policy. 2010;2(4):311-7.

56. Lopez EJ, Jones DL, Villar-Loubet OM, Arheart KL, Weiss SM. Violence, coping, and consistent medication adherence in HIV-positive couples. AIDS Educ and Prev : Official Publication of the International Society for AIDS Education. 2010;22(1):61-8.

57. Espino SR, Fletcher J, Gonzalez M, Precht A, Xavier J, Matoff-Stepp S. Violence screening and viral load suppression among HIV-positive women of color. AIDS Patient Care STDs. 2015;29(Suppl 1):S36-41.

58. Kidman R, Violari A. Dating violence against HIV-infected youth in South Africa: associations with sexual risk behavior, medication adherence, and mental health. J Acquir Immune Defic Syndr. 2018;77(1):64-71.

59. Kacanek D, Malee K, Mellins CA, Tassiopoulos K, Smith R, Grant M, et al. Exposure to violence and Virologic and immunological outcomes among youth with perinatal HIV in the pediatric HIV/AIDS cohort study. J Adolesc Health. 2016;59(1):30-7.

60. Cohen MH, Cook JA, Grey D, Young M, Hanau LH, Tien P, et al. Medically eligible women who do not use HAART: the importance of abuse, drug use, and race. Am J Public Health. 2004;94(7):1147-51.

61. Mendoza C, Barrington C, Donastorg Y, Perez M, Fleming PJ, Decker MR, et al. Violence from a sexual partner is significantly associated with poor HIV care and treatment outcomes among female sex Workers in the Dominican Republic. J Acquir Immune Defic Syndr. 2017;74(3):273-8.

62. Lyons C, Ketende S, Drame F, Grosso A, Diouf D, Ba I, et al. Physical and sexual violence affecting female sex workers in Abidjan, cote d'lvoire: prevalence, and the relationship between violence, the work environment, HIV and access to health services. J Acquir Immune Defic Syndr. 2017;75(1):9-17.

63. Machtinger EL, Haberer JE, Wilson TC, Weiss DS. Recent trauma is associated with antiretroviral failure and HIV transmission risk behavior among HIV-positive women and female-identified transgenders. AIDS Behav. 2012;16(8):2160-70.

64. Kalokhe AS, Paranjape A, Bell CE, Cardenas GA, Kuper T, Metsch LR, et al. Intimate partner violence among HIV-infected crack cocaine users. AIDS Patient Care STDs. 2012;26(4):234-40.
65. Wechsberg WM, van der Horst C, Ndirangu J, Doherty IA, Kline T, Browne FA, et al. Seek, test, treat: substance-using women in the HIV treatment cascade in South Africa. Addiction science \& clinical practice. 2017:12(1):12-017-0077-x.

66. Mepham S, Zondi Z, Mbuyazi A, Mkhwanazi N, Newell ML. Challenges in PMTCT antiretroviral adherence in northern KwaZulu-Natal, South Africa. AIDS Care. 2011;23(6):741-7.

67. Zunner B, Dworkin SL, Neylan TC, Bukusi EA, Oyaro P, Cohen CR, et al. $H I V$, violence and women: unmet mental health care needs. J Affect Disord. 2015;174:619-26.

68. Hatcher AM, Stockl H, Christofides N, Woollett N, Pallitto CC, Garcia-Moreno $\mathrm{C}$, et al. Mechanisms linking intimate partner violence and prevention of mother-to-child transmission of HIV: a qualitative study in South Africa. Social science \& medicine (1982). 2016;168:130-9.

69. Conroy A, Leddy A, Johnson M, Ngubane T, van Rooyen H, Darbes L. 'I told her this is your life': relationship dynamics, partner support and adherence to antiretroviral therapy among south African couples. Culture, health \& sexuality. 2017;19(11):1239-53.

70. Lichtenstein B. Domestic violence in barriers to health care for HIV-positive women. AIDS Patient Care STDs. 2006;20(2):122-32.

71. Watt MH, Dennis AC, Choi KW, Ciya N, Joska JA, Robertson C, et al. Impact of sexual trauma on HIV care engagement: perspectives of female patients with trauma histories in Cape Town, South Africa. AIDS Behav. 2017;21(11):3209-18.

72. Kosia A, Kakoko D, Semakafu AM, Nyamhanga T, Frumence G. Intimate partner violence and challenges facing women living with HIV/AIDS in accessing antiretroviral treatment at Singida regional hospital, Central Tanzania. Glob Health Action. 2016;9:32307.

73. Maeri I, El Ayadi A, Getahun M, Charlebois E, Akatukwasa C, Tumwebaze D, et al. "how can I tell?" consequences of HIV status disclosure among couples in eastern African communities in the context of an ongoing HIV "test-and-treat" trial. AIDS Care. 2016;28(Suppl 3):59-66.

74. Orza LB, E.; Bell, E.; Crone, E.T.; Damji, N.; Dilmitis, S.; Tremlett, L.; Aidarus, N.; Stevenson, J.; Bensaid, S.; Kenkem, C.; Ross, G.V.; Kudravtseva, E.; Welbourn, A. In women's eyes: key barriers to women's access to HIV treatment and a rights-based approach to their sutstained well-being. Health and Human Rights Journal 2017;19(2):155-168.

75. Wilson KS, Wanje G, Yuhas K, Simoni JM, Masese L, Vander Stoep A, et al. A prospective study of intimate partner violence as a risk factor for detectable plasma viral load in HIV-positive women engaged in transactional sex in Mombasa, Kenya. AIDS Behav. 2016;20(9):2065-77.

76. Roberts ST, Haberer J, Celum C, Mugo N, Ware NC, Cohen CR, et al. Intimate partner violence and adherence to HIV pre-exposure prophylaxis (PrEP) in African women in HIV serodiscordant relationships: a prospective cohort study. J Acquir Immune Defic Syndr. 2016;73(3):313-22.

77. Raj A, Silverman JG, Amaro H. Abused women report greater male partner risk and gender-based risk for HIV: findings from a community-based study with Hispanic women. AIDS Care. 2004;16(4):519-29.

78. Reisner SL, Poteat T, Keatley J, Cabral M, Mothopeng T, Dunham E, et al. Global health burden and needs of transgender populations: a review. Lancet. 2016;338:412-36.

79. Collins DA, Shamblen SR, Strader TN, Arnold BB. Evaluation of an evidencebased intervention implemented with African-American women to prevent substance abuse, strengthen relationship skills and reduce risk for HIV/AIDS. AIDS Care. 2017;29(8):966-73.

80. Abramsky T, Devries KM, Michau L, Nakuti J, Musuya T, Kyegombe N, et al. The impact of SASA!, a community mobilisation intervention, on women's experiences of intimate partner violence: secondary findings from a cluster randomised trial in Kampala, Uganda. J Epidemiol Community Health. 2016;70(8):818-25.

81. Jewkes R, Nduna M, Levin J, Jama N, Dunkle K, Puren A, et al. Impact of stepping stones on incidence of HIV and HSV-2 and sexual behaviour in rural South Africa: cluster randomised controlled trial. BMJ (Clinical research ed). 2008;337:a506.

82. Wagman JA, Gray RH, Campbell JC, Thoma M, Ndyanabo A, Ssekasanvu J, et al. Effectiveness of an integrated intimate partner violence and HIV prevention intervention in Rakai, Uganda: analysis of an intervention in an existing cluster randomised cohort. The LancetGlobal health. 2015;3(1):e23-33.

83. Pulerwitz J, Hughes L, Mehta M, Kidanu A, Verani F, Tewolde S. Changing gender norms and reducing intimate partner violence: results from a quasi-experimental intervention study with Young men in Ethiopia. Am J Public Health. 2015;105(1):132-7. 
84. Pulerwitz JB, G.; Verma, R. Changing gender norms for HIV and violence risk reduction. In: Obergon RW, S., editor. The Handbook of Global Health Communication. Oxford: Wiley-Blackwell; 2012.

85. Christofides N, Jewkes R. Acceptability of universal screening for intimate partner violence in voluntary HIV testing and counseling services in South Africa and service implications. AIDS Care. 2010;22(3):279-85.

86. Undie CC, Maternowska MC, Mak'anyengo M, Askew I. Is routine screening for intimate partner violence feasible in public Health care settings in Kenya. Journal of Interpersonal Violence. 2016;31(2):282-301.

\section{Publisher's Note}

Springer Nature remains neutral with regard to jurisdictional claims in published maps and institutional affiliations.

Ready to submit your research? Choose BMC and benefit from:

- fast, convenient online submission

- thorough peer review by experienced researchers in your field

- rapid publication on acceptance

- support for research data, including large and complex data types

- gold Open Access which fosters wider collaboration and increased citations

- maximum visibility for your research: over $100 \mathrm{M}$ website views per year

At $\mathrm{BMC}$, research is always in progress.

Learn more biomedcentral.com/submissions 